\title{
On the Difficulty of Deciding Asymptotic Stability of Cubic Homogeneous Vector Fields
}

\author{
Amir Ali Ahmadi
}

\begin{abstract}
It is well-known that asymptotic stability (AS) of homogeneous polynomial vector fields of degree one (i.e., linear systems) can be decided in polynomial time e.g. by searching for a quadratic Lyapunov function. Since homogeneous vector fields of even degree can never be AS, the next interesting degree to consider is equal to three. In this paper, we prove that deciding AS of homogeneous cubic vector fields is strongly NP-hard and pose the question of determining whether it is even decidable. As a byproduct of the reduction that establishes our NP-hardness result, we obtain a Lyapunov-inspired technique for proving positivity of forms. We also show that for asymptotically stable homogeneous cubic vector fields in as few as two variables, the minimum degree of a polynomial Lyapunov function can be arbitrarily large. Finally, we show that there is no monotonicity in the degree of polynomial Lyapunov functions that prove AS; i.e., a homogeneous cubic vector field with no homogeneous polynomial Lyapunov function of some degree $d$ can very well have a homogeneous polynomial Lyapunov function of degree less than $d$.
\end{abstract}

\section{INTRODUCTION}

\section{A. Background}

We are concerned in this paper with a continuous time dynamical system

$$
\dot{x}=f(x),
$$

where $f: \mathbb{R}^{n} \rightarrow \mathbb{R}^{n}$ is a polynomial and has an equilibrium at the origin, i.e., $f(0)=0$. Polynomial differential equations appear ubiquitously in engineering and sciences either as true models of physical systems, or as approximations to other families of nonlinear dynamics. The problem of deciding stability of equilibrium points of such systems is of fundamental importance in control theory. The goal of this paper is to demonstrate some of the difficulties associated with answering stability questions about polynomial vector fields in terms of both computational complexity and nonexistence of "simple" Lyapunov functions, even if one limits attention to very restricted settings.

The notion of stability of interest in this paper is (local or global) asymptotic stability. The origin of (1) is said to be stable in the sense of Lyapunov if for every $\epsilon>0$, there exists a $\delta=\delta(\epsilon)>0$ such that

$$
\|x(0)\|<\delta \Rightarrow\|x(t)\|<\epsilon, \quad \forall t \geq 0 .
$$

We say that the origin is asymptotically stable (AS) if it is stable in the sense of Lyapunov and $\delta$ can be chosen such that

$$
\|x(0)\|<\delta \Rightarrow \lim _{t \rightarrow \infty} x(t)=0 .
$$

Amir Ali Ahmadi is with the Laboratory for Computer Science and Artificial Intelligence, Department of Electrical Engineering and Computer Science, Massachusetts Institute of Technology. E-mail: a_a_a@mit.edu.
The origin is globally asymptotically stable (GAS) if it is stable in the sense of Lyapunov and $\forall x(0) \in \mathbb{R}^{n}$, $\lim _{t \rightarrow \infty} x(t)=0$.

The degree of the vector field in (1) is defined to be the largest degree of the components of $f$. Our focus in this paper is on homogeneous polynomial vector fields. A scalar valued function $p: \mathbb{R}^{n} \rightarrow \mathbb{R}$ is said to be homogeneous (of degree $d$ ) if it satisfies $p(\lambda x)=\lambda^{d} p(x)$ for all $x \in \mathbb{R}^{n}$ and all $\lambda \in \mathbb{R}$. A homogeneous polynomial is also called a form. All monomials of a form share the same degree. We say that the vector field $f$ in (1) is homogeneous if all components of $f$ are forms of the same degree. Homogeneous systems are extensively studied in the literature on nonlinear control; see e.g. [1], [2], [3], [4], [5], [6], [7]. Since our results are negative in nature, their validity for homogeneous polynomial systems obviously also implies their validity for all polynomial systems.

A basic fact about homogeneous vector fields is that for these systems the notions of local and global asymptotic stability are equivalent. Indeed, a homogeneous vector field of degree $d$ satisfies $f(\lambda x)=\lambda^{d} f(x)$ for any scalar $\lambda$, and therefore the value of $f$ on the unit sphere determines its value everywhere. It is also well-known that an asymptotically stable homogeneous system admits a homogeneous Lyapunov function [8, Sec. 57], [6].

\section{B. An open question of Arnold}

It is natural to ask whether stability of equilibrium points of polynomial vector fields can be decided in finite time. In fact, this is a well-known question of Arnold that appears in [9]:

"Is the stability problem for stationary points algorithmically decidable? The well-known Lyapunov theorem 1 solves the problem in the absence of eigenvalues with zero real parts. In more complicated cases, where the stability depends on higher order terms in the Taylor series, there exists no algebraic criterion.

Let a vector field be given by polynomials of a fixed degree, with rational coefficients. Does an algorithm exist, allowing to decide, whether the stationary point is stable?"

To our knowledge, there has been no formal resolution to this question, neither for the case of stability in the sense of Lyapunov, nor for the case of asymptotic stability (in its local or global version). In [10], da Costa and Doria

${ }^{1}$ The theorem that Arnold is referring to here is the indirect method of Lyapunov related to linearization. This is not to be confused with Lyapunov's direct method (or the second method), which is what we are concerned with in sections that follow. 
show that if the right hand side of the differential equation contains elementary functions (sines, cosines, exponentials, absolute value function, etc.), then there is no algorithm for deciding whether the origin is stable or unstable. They also present a dynamical system in [11] where one cannot decide whether a Hopf bifurcation will occur or whether there will be parameter values such that a stable fixed point becomes unstable. A relatively larger number of undecidability results are available for questions related to other properties of polynomial vector fields, such as reachability [12] or boundedness of domain of definition [13], or for questions about stability of hybrid systems [14], [15], [16], [17]. We refer the interested reader to the survey papers in [18], [12], [19], [20], [21].

We are also interested to know whether the problem of deciding asymptotic stability of homogeneous polynomial vector fields is undecidable for some fixed degree, say, equal to 3 . The answer to such decidability questions, or at least the level of difficulty associated with proving such results, can depend in a subtle way on the exact criteria in question. For example, it has been known for a while that the question of determining boundedness of trajectories for arbitrarily switched linear systems is undecidable [15] even when one restricts attention to switched systems defined by nonnegative matrices. On the other hand, the complexity of testing asymptotic stability for the same class of systems remains open and in fact is conjectured to be decidable [22].

\section{Existence of polynomial Lyapunov functions}

For stability analysis of polynomial vector fields, it is most common (and quite natural) to search for Lyapunov functions that are polynomials themselves. This approach has become further prevalent over the past decade due to the fact that techniques from sum of squares optimization [23] have provided for algorithms that given a polynomial system can efficiently search for a polynomial Lyapunov function [23], [24]. The question is therefore naturally motivated to determine whether stable polynomial systems always admit polynomial Lyapunov functions, and whether one can give upper bounds on the degree of such Lyapunov functions in cases when they do exist. A study of questions of this type for different notions of stability has recently been carried out in [25], [26], [27], [28], [29, Chap. 4]. In this paper, we continue this line of research by studying the case where the vector field is homogeneous.

Throughout this paper, by a (polynomial) Lyapunov function for (1), we mean a positive definite polynomial function $V$ whose derivatives $\dot{V}$ along trajectories of (1) is negative definite; i.e., a function $V$ satisfying

$$
\begin{array}{r}
V(x)>0 \quad \forall x \neq 0 \\
\dot{V}(x)=\langle\nabla V(x), f(x)\rangle<0 \quad \forall x \neq 0 .
\end{array}
$$

Here, $\nabla V(x)$ denotes the gradient vector of $V$, and $\langle.,$. is the standard inner product in $\mathbb{R}^{n}$. If such a $V$ is also radially unbounded, then the inequalities in (2) and (3) imply that the origin of (1) is GAS. When the dynamics $f$ is homogeneous, we can restrict our search to homogeneous polynomials. Such a Lyapunov function is automatically radially unbounded and proves (local or equivalently global) asymptotic stability of the homogeneous vector field.

Naturally, questions regarding complexity of deciding asymptotic stability and questions about existence of Lyapunov functions are related. For instance, if one proves that for a class of polynomial vector fields, asymptotic stability implies existence of a polynomial Lyapunov function together with a computable upper bound on its degree, then the question of asymptotic stability for that class becomes decidable. This is due to the fact that given a polynomial system and an integer $d$, the question of deciding whether the system admits a polynomial Lyapunov function of degree $d$ can be answered in finite time using quantifier elimination [30], [31].

For the case of linear systems (i.e., homogeneous systems of degree 1), the situation is particularly nice. If such a system is asymptotically stable, then there always exists a quadratic Lyapunov function. Asymptotic stability of a linear system $\dot{x}=A x$ is equivalent to the easily checkable algebraic criterion that the eigenvalues of $A$ be in the open left half complex plane. Deciding this property of the matrix $A$ can formally be done in polynomial time, e.g. by solving a Lyapunov equation [21].

Moving up in the degree, it is not difficult to show that if a homogeneous polynomial vector field has even degree, then it can never be asymptotically stable; see e.g. [8, p. 283]. So the next interesting case occurs for homogeneous vector fields of degree 3 . We will prove three results in this paper which demonstrate that already for cubic homogeneous systems, the situation is significantly more complex than it is for linear systems. We outline our contributions next.

\section{Contributions and organization of this paper}

In Section II we prove that determining asymptotic stability for homogeneous cubic vector fields is strongly NP-hard (Theorem 2.1. Although this of course does not resolve the question of Arnold, the result gives a lower bound on the complexity of this problem. It is an interesting open question to investigate whether in this specific setting, the problem is also undecidable.

The implication of the NP-hardness of this problem is that unless $\mathrm{P}=\mathrm{NP}$, it is impossible to design an algorithm that can take as input the (rational) coefficients of a homogeneous cubic vector field, have running time bounded by a polynomial in the number of bits needed to represent these coefficients, and always output the correct yes/no answer on asymptotic stability. Moreover, the fact that our NP-hardness result is in the strong sense (as opposed to weakly NP-hard problems such as KNAPSACK, SUBSET SUM, etc.) implies that the problem remains NP-hard even if the size (bit length) of the coefficients is $O(\log n)$, where $n$ is the dimension. For a strongly NP-hard problem, even a pseudo-polynomial time algorithm cannot exist unless $\mathrm{P}=\mathrm{NP}$. See [32] for precise definitions and more details.

In Section II, we also present a Lyapunov-inspired technique for proving positivity of forms that comes directly 
out of the reduction in the proof of our NP-hardness result (Corollary 2.1). We show the potential advantages of this technique over standard sum of squares techniques on an example (Example 2.1).

In Section III, we prove that unlike AS linear systems that always admit quadratic Lyapunov functions, AS cubic homogeneous systems may need polynomial Lyapunov functions of arbitrarily large degree, even when the dimension is fixed to 2 (Theorem 3.1). Finally, in Section IV we show that there is no monotonicity in the degree of homogeneous polynomial Lyapunov functions for homogeneous cubic vector fields. We give an example of such a vector field which admits a homogeneous polynomial Lyapunov function of degree 4 but not one of degree 6 (Theorem 4.1).

\section{NP-HARDNESS OF DECIDING ASYMPTOTIC STABILITY OF HOMOGENEOUS CUBIC VECTOR FIELDS}

The main result of this section is the following theorem.

Theorem 2.1: Deciding asymptotic stability of homogeneous cubic polynomial vector fields is strongly NP-hard.

The key idea behind the proof of this theorem is the following: We will relate the solution of a combinatorial problem not to the behavior of the trajectories of a cubic vector field that are hard to get a handle on, but instead to properties of a Lyapunov function that proves asymptotic stability of this vector field. As we will see shortly, insights from Lyapunov theory make the proof of this theorem quite simple. The reduction is broken into two steps:

\author{
ONE-IN-THREE 3SAT \\ $\downarrow$ \\ positivity of quartic forms \\ $\downarrow$ \\ asymptotic stability of cubic vector fields
}

\section{A. Reduction from ONE-IN-THREE 3SAT to positivity of quartic forms}

A form $q$ is said to be nonnegative or positive semidefinite if $q(x) \geq 0$ for all $x$ in $\mathbb{R}^{n}$. We say that a form $q$ is positive definite if $q(x)>0$ for all $x \neq 0$ in $\mathbb{R}^{n}$. (Note that forms necessarily vanish at the origin.) It is well-known that deciding nonnegativity of quartic forms is NP-hard; see e.g. [33] and [34]. For reasons that will become clear shortly, we are interested instead in showing hardness of deciding positive definiteness of quartic forms. This is in some sense even easier to accomplish. A very straightforward reduction from 3SAT proves NP-hardness of deciding positive definiteness of polynomials of degree 6 . By using ONE-IN-THREE 3SAT instead, we will reduce the degree of the polynomial from 6 to 4 .

Proposition 1: It is strongly 2 NP-hard to decide whether a homogeneous polynomial of degree 4 is positive definite.

Proof: We give a reduction from ONE-IN-THREE 3SAT which is known to be NP-complete [32, p. 259]. Recall that in ONE-IN-THREE 3SAT, we are given a 3SAT instance

\footnotetext{
${ }^{2}$ The NP-hardness results of this section will all be in the strong sense. From here on, we drop the prefix "strong" for brevity.
}

(i.e., a collection of clauses, where each clause consists of exactly three literals, and each literal is either a variable or its negation) and we are asked to decide whether there exists a $\{0,1\}$ assignment to the variables that makes the expression true with the additional property that each clause has exactly one true literal.

To avoid introducing unnecessary notation, we present the reduction on a specific instance. The pattern will make it obvious that the general construction is no different. Given an instance of ONE-IN-THREE 3SAT, such as the following

$\left(x_{1} \vee \bar{x}_{2} \vee x_{4}\right) \wedge\left(\bar{x}_{2} \vee \bar{x}_{3} \vee x_{5}\right) \wedge\left(\bar{x}_{1} \vee x_{3} \vee \bar{x}_{5}\right) \wedge\left(x_{1} \vee x_{3} \vee x_{4}\right)$,

we define the quartic polynomial $p$ as follows:

$$
\begin{aligned}
p(x)= & \sum_{i=1}^{5} x_{i}^{2}\left(1-x_{i}\right)^{2} \\
& +\left(x_{1}+\left(1-x_{2}\right)+x_{4}-1\right)^{2}+\left(\left(1-x_{2}\right)\right. \\
& \left.+\left(1-x_{3}\right)+x_{5}-1\right)^{2} \\
& +\left(\left(1-x_{1}\right)+x_{3}+\left(1-x_{5}\right)-1\right)^{2} \\
& +\left(x_{1}+x_{3}+x_{4}-1\right)^{2} .
\end{aligned}
$$

Having done so, our claim is that $p(x)>0$ for all $x \in \mathbb{R}^{5}$ (or generally for all $x \in \mathbb{R}^{n}$ ) if and only if the ONE-INTHREE 3SAT instance is not satisfiable. Note that $p$ is a sum of squares and therefore nonnegative. The only possible locations for zeros of $p$ are by construction among the points in $\{0,1\}^{5}$. If there is a satisfying Boolean assignment $x$ to (4) with exactly one true literal per clause, then $p$ will vanish at point $x$. Conversely, if there are no such satisfying assignments, then for any point in $\{0,1\}^{5}$, at least one of the terms in (5) will be positive and hence $p$ will have no zeros.

It remains to make $p$ homogeneous. This can be done via introducing a new scalar variable $y$. If we let

$$
p_{h}(x, y)=y^{4} p\left(\frac{x}{y}\right),
$$

then we claim that $p_{h}$ (which is a quartic form) is positive definite if and only if $p$ constructed as in (5) has no zeros 3 Indeed, if $p$ has a zero at a point $x$, then that zero is inherited by $p_{h}$ at the point $(x, 1)$. If $p$ has no zeros, then (6) shows that $p_{h}$ can only possibly have zeros at points with $y=0$. However, from the structure of $p$ in (5) we see that

$$
p_{h}(x, 0)=x_{1}^{4}+\cdots+x_{5}^{4},
$$

which cannot be zero (except at the origin). This concludes the proof.

B. Reduction from positivity of quartic forms to asymptotic stability of cubic vector fields

We now present the second step of the reduction and finish the proof of Theorem 2.1.

\footnotetext{
${ }^{3}$ In general, the homogenization operation in 6) does not preserve positivity. For example, as shown in [35], the polynomial $x_{1}^{2}+\left(1-x_{1} x_{2}\right)^{2}$ has no zeros, but its homogenization $x_{1}^{2} y^{2}+\left(y^{2}-x_{1} x_{2}\right)^{2}$ has zeros at the points $(1,0,0)^{T}$ and $(0,1,0)^{T}$. Nevertheless, positivity is preserved under homogenization for the special class of polynomials constructed in this reduction, essentially because polynomials of type 5 have no zeros at infinity.
} 
Proof: [Proof of Theorem 2.1] We give a reduction from the problem of deciding positive definiteness of quartic forms, whose NP-hardness was established in Proposition 1 Given a quartic form $V:=V(x)$, we define the polynomial vector field

$$
\dot{x}=-\nabla V(x) .
$$

Note that the vector field is homogeneous of degree 3 . We claim that the above vector field is (locally or equivalently globally) asymptotically stable if and only if $V$ is positive definite. First, we observe that by construction

$$
\dot{V}(x)=\langle\nabla V(x), \dot{x}\rangle=-\|\nabla V(x)\|^{2} \leq 0 .
$$

Suppose $V$ is positive definite. By Euler's identity for homogeneous functions 4 we have $V(x)=\frac{1}{4} x^{T} \nabla V(x)$. Therefore, positive definiteness of $V$ implies that $\nabla V(x)$ cannot vanish anywhere except at the origin. Hence, $\dot{V}(x)<0$ for all $x \neq 0$. In view of Lyapunov's theorem (see e.g. [36, p. 124]), and the already mentioned fact that a positive definite homogeneous function is radially unbounded, it follows that the system in (7) is globally asymptotically stable.

For the converse direction, suppose (7) is GAS. Our first claim is that global asymptotic stability together with $\dot{V}(x) \leq 0$ implies that $V$ must be positive semidefinite. This follows from the following simple argument, which we have also previously presented in [37] for a different purpose. Suppose for the sake of contradiction that for some $\hat{x} \in \mathbb{R}^{n}$ and some $\epsilon>0$, we had $V(\hat{x})=-\epsilon<0$. Consider a trajectory $x(t ; \hat{x})$ of system (7) that starts at initial condition $\hat{x}$, and let us evaluate the function $V$ on this trajectory. Since $V(\hat{x})=-\epsilon$ and $\dot{V}(x) \leq 0$, we have $V(x(t ; \hat{x})) \leq-\epsilon$ for all $t>0$. However, this contradicts the fact that by global asymptotic stability, the trajectory must go to the origin, where $V$, being a form, vanishes.

To prove that $V$ is positive definite, suppose by contradiction that for some nonzero point $x^{*} \in \mathbb{R}^{n}$ we had $V\left(x^{*}\right)=0$. Since we just proved that $V$ has to be positive semidefinite, the point $x^{*}$ must be a global minimum of $V$. Therefore, as a necessary condition of optimality, we should have $\nabla V\left(x^{*}\right)=0$. But this contradicts the system in (7) being GAS, since the trajectory starting at $x^{*}$ stays there forever and can never go to the origin.

Perhaps of independent interest, the reduction we just gave suggests a method for proving positive definiteness of forms. Given a form $V$, we can construct a dynamical system as in 17, and then any method that we may have for proving stability of vector fields (e.g. the use of various kinds of Lyapunov functions) can serve as an algorithm for proving positivity of $V$. In particular, if we use a polynomial Lyapunov function $W$ to prove stability of the system in 7 , we get the following corollary.

Corollary 2.1: Let $V$ and $W$ be two forms of possibly different degree. If $W$ is positive definite, and $\langle\nabla W, \nabla V\rangle$ is positive definite, then $V$ is positive definite.

\footnotetext{
${ }^{4}$ Euler's identity is easily derived by differentiating both sides of the equation $V(\lambda x)=\lambda^{d} V(x)$ with respect to $\lambda$ and setting $\lambda=1$.
}

A polynomial $p$ is said to be a sum of squares (sos) if it can be written as $p=\sum_{i=1}^{m} q_{i}^{2}$ for some polynomials $q_{i}$. An sos polynomial is clearly nonnegative. Moreover, unlike the property of nonnegativity that is NP-hard to check, existence of an sos decomposition can be cast as a semidefinite program [38], which can be solved efficiently. However, not every nonnegative polynomial is a sum of squares.

An interesting fact about Corollary 2.1 is that its algebraic version with sum of squares replaced for positivity is not true. In other words, we can have $W$ sos (and positive definite), $\langle\nabla W, \nabla V\rangle$ sos (and positive definite), but $V$ not sos. This gives us a way of proving positivity of some polynomials that are not sos, using only sos certificates. Given a form $V$, since the expression $\langle\nabla W, \nabla V\rangle$ is linear in the coefficients of $W$, we can use semidefinite programming to search for a form $W$ that satisfies $W$ sos and $\langle\nabla W, \nabla V\rangle$ sos, and this would prove positivity of $V$. The following example demonstrates the potential usefulness of this approach.

Example 2.1: Consider the following form of degree 6:

$V(x)=x_{1}^{4} x_{2}^{2}+x_{1}^{2} x_{2}^{4}-3 x_{1}^{2} x_{2}^{2} x_{3}^{2}+x_{3}^{6}+\frac{1}{250}\left(x_{1}^{2}+x_{2}^{2}+x_{3}^{2}\right)^{3}$.

One can check that this polynomial is not a sum of squares. (In fact, this is the celebrated Motzkin form [39] slightly perturbed.) On the other hand, we can use the software package YALMIP [40] together with the SDP solver SeDuMi [41] to search for a form $W$ satisfying

$$
\begin{aligned}
W & \text { sos } \\
\langle\nabla W, \nabla V\rangle & \text { sos. }
\end{aligned}
$$

If we parameterize $W$ as a quadratic form, no feasible solution will be returned form the solver. However, when we increase the degree of $W$ from 2 to 4 , the solver returns the following polynomial

$$
\begin{aligned}
W(x)= & 9 x_{2}^{4}+9 x_{1}^{4}-6 x_{1}^{2} x_{2}^{2}+6 x_{1}^{2} x_{3}^{2}+6 x_{2}^{2} x_{3}^{2}+3 x_{3}^{4} \\
& -x_{1}^{3} x_{2}-x_{1} x_{2}^{3}-x_{1}^{3} x_{3}-3 x_{1}^{2} x_{2} x_{3}-3 x_{1} x_{2}^{2} x_{3} \\
& -x_{2}^{3} x_{3}-4 x_{1} x_{2} x_{3}^{2}-x_{1} x_{3}^{3}-x_{2} x_{3}^{3}
\end{aligned}
$$

that satisfies both sos constraints in (10). One can easily infer from the sos decompositions (e.g. by checking positive definiteness of the associated "Gram matrices") that the forms $W$ and $\langle\nabla W, \nabla V\rangle$ are positive definite. Hence, by Corollary 2.1. we have a proof that $V$ in (9) is positive definite. $\triangle$

Interestingly, approaches of this type that use gradient information for proving positivity of polynomials with sum of squares techniques have been studied by Nie, Demmel, and Sturmfels in [42], though the derivation there is not inspired by Lyapunov theory.

\section{NON-EXISTENCE OF A UNIFORM BOUND ON THE DEGREE OF POLYNOMIAL LYAPUNOV FUNCTIONS IN FIXED DIMENSION AND DEGREE}

For polynomial vector fields in general, existence of a polynomial Lyapunov function is not necessary for global asymptotic stability. In joint work with M. Krstic and P.A. Parrilo [27], we recently gave a remarkably simple example 
of a (non-homogeneous) quadratic polynomial vector field in two variables that is GAS but does not admit a polynomial Lyapunov function (of any degree). An independent earlier example that appears in a book by Bacciotti and Rosier [43, Prop. 5.2] was brought to our attention after our work was submitted. We refer the reader to [27] for a discussion on the differences between the two examples, the main one being that the example in [43] does not admit a polynomial Lyapunov function even locally but unlike the example in [27] relies on using irrational coefficients.

The situation for homogeneous polynomial vector fields, however, seems to be different. We conjecture that for such systems, existence of a homogeneous polynomial Lyapunov function is necessary and sufficient for (global) asymptotic stability. The reason for this conjecture is that we expect that one should be able to approximate a continuously differentiable Lyapunov function with a polynomial one on the unit sphere, which by homogeneity should be enough to imply the Lyapunov inequalities everywhere. A formal treatment of this idea is left for future work. Here, we build on the result in [43, Prop. 5.2] to prove that the minimum degree of a polynomial Lyapunov function for an AS homogeneous vector field can be arbitrarily large even when the degree and dimension are fixed respectively to 3 and 2 . field

Proposition 2 ( [43, Prop. 5.2-a]): Consider the vector

$$
\begin{aligned}
& \dot{x}=-2 \lambda y\left(x^{2}+y^{2}\right)-2 y\left(2 x^{2}+y^{2}\right) \\
& \dot{y}=4 \lambda x\left(x^{2}+y^{2}\right)+2 x\left(2 x^{2}+y^{2}\right)
\end{aligned}
$$

parameterized by the scalar $\lambda>0$. For all values of $\lambda$ the origin is a center for (11), but for any irrational value of $\lambda$ there exists no polynomial function $V$ satisfying $\dot{V}(x, y)=$ $\frac{\partial V}{\partial x} \dot{x}+\frac{\partial V}{\partial y} \dot{y}=0$.

Theorem 3.1: Let $\lambda$ be a positive irrational real number and consider the following homogeneous cubic vector field parameterized by the scalar $\theta$ :

$$
\left(\begin{array}{l}
\dot{x} \\
\dot{y}
\end{array}\right)=\left(\begin{array}{rr}
\cos (\theta) & -\sin (\theta) \\
\sin (\theta) & \cos (\theta)
\end{array}\right)\left(\begin{array}{r}
-2 \lambda y\left(x^{2}+y^{2}\right)-2 y\left(2 x^{2}+y^{2}\right) \\
4 \lambda x\left(x^{2}+y^{2}\right)+2 x\left(2 x^{2}+y^{2}\right)
\end{array}\right) .
$$

Then for any even degree $d$ of a candidate polynomial Lyapunov function, there exits a $\theta>0$ small enough such that the vector field in 12 is asymptotically stable but does not admit a polynomial Lyapunov function of degree $\leq d$.

Proof: Consider the (non-polynomial) positive definite Lyapunov function

$$
V(x, y)=\left(2 x^{2}+y^{2}\right)^{\lambda}\left(x^{2}+y^{2}\right)
$$

whose derivative along the trajectories of 12 is equal to

$$
\dot{V}(x, y)=-\sin (\theta)\left(2 x^{2}+y^{2}\right)^{\lambda-1}\left(\dot{x}^{2}+\dot{y}^{2}\right) .
$$

Since $\dot{V}$ is negative definite for $0<\theta<\pi$, it follows that for $\theta$ in this range, the origin of (12) is asymptotically stable.

To establish the claim in the theorem, suppose for the sake of contradiction that there exists an upper bound $\bar{d}$ such that for all $0<\theta<\pi$ the system admits a (homogeneous) polynomial Lyapunov function of degree $d(\theta)$ with $d(\theta) \leq \bar{d}$. Let $\hat{d}$ be the least common multiplier of the degrees $\bar{d}(\theta)$ for $0<\theta<\pi$. (Note that $d(\theta)$ can at most range over all even positive integers less than or equal to $\bar{d}$.) Since positive powers of Lyapunov functions are valid Lyapunov functions, it follows that for every $0<\theta<\pi$, the system admits a homogeneous polynomial Lyapunov function $W_{\theta}$ of degree $\hat{d}$. By rescaling, we can assume without loss of generality that all Lyapunov functions $W_{\theta}$ have unit area on the unit sphere. Let us now consider the sequence $\left\{W_{\theta}\right\}$ as $\theta \rightarrow 0$. We think of this sequence as residing in a compact subset of $\mathbb{R}^{\left(\begin{array}{c}\hat{d}+1 \\ \hat{d}\end{array}\right)}$ associated with the set $P_{2, \hat{d}}$ of (coefficients of) all nonnegative bivariate homogeneous polynomials of degree $\hat{d}$ with unit area on the unit sphere. Since every bounded sequence has a converging subsequence, it follows that there must exist a subsequence of $\left\{W_{\theta}\right\}$ that converges (in the coefficient sense) to some polynomial $W_{0}$ belonging to $P_{2, \hat{d}}$. Since convergence of this subsequence also implies convergence of the associated gradient vectors, we get that

$$
\dot{W}_{0}(x, y)=\frac{\partial W_{0}}{\partial x} \dot{x}+\frac{\partial W_{0}}{\partial y} \dot{y} \leq 0 .
$$

On the other hand, when $\theta=0$, the vector field in (12) is the same as the one in 111 and hence the trajectories starting from any nonzero initial condition go on periodic orbits. This however implies that $\dot{W}=0$ everywhere and in view of Proposition 2 we have a contradiction.

Remark 3.1: Unlike the result in [43, Prop. 5.2], it is easy to establish the result of Theorem 3.1 without having to use irrational coefficients in the vector field. One approach is to take an irrational number, e.g. $\pi$, and then think of a sequence of vector fields given by 12 that is parameterized by both $\theta$ and $\lambda$. We let the $k$-th vector field in the sequence have $\theta_{k}=\frac{1}{k}$ and $\lambda_{k}$ equal to a rational number representing $\pi$ up to $k$ decimal digits. Since in the limit as $k \rightarrow \infty$ we have $\theta_{k} \rightarrow 0$ and $\lambda_{k} \rightarrow \pi$, it should be clear from the proof of Theorem 3.1 that for any integer $d$, there exists an AS bivariate homogeneous cubic vector field with rational coefficients that does not have a polynomial Lyapunov function of degree less than $d$.

\section{LACK OF MONOTONICITY IN THE DEGREE OF POLYNOMIAL LYAPUNOV FUNCTIONS}

If a dynamical system admits a quadratic Lyapunov function $V$, then it clearly also admits a polynomial Lyapunov function of any higher even degree (e.g. simply given by $V^{k}$ for $\left.k=2,3, \ldots\right)$. However, our next theorem shows that for homogeneous systems that do not admit a quadratic Lyapunov function, such a monotonicity property in the degree of polynomial Lyapunov functions may not hold.

Theorem 4.1: Consider the following homogeneous cubic vector field parameterized by the scalar $\theta$ :

$$
\left(\begin{array}{l}
\dot{x} \\
\dot{y}
\end{array}\right)=\left(\begin{array}{rr}
-\sin (\theta) & \cos (\theta) \\
-\cos (\theta) & -\sin (\theta)
\end{array}\right)\left(\begin{array}{l}
x^{3} \\
y^{3}
\end{array}\right) .
$$

There exists a range of values for the parameter $\theta>0$ for which the vector field is asymptotically stable, has no homogeneous polynomial Lyapunov function of degree 6 , 
but admits a homogeneous polynomial Lyapunov function of degree 4.

Proof: Consider the positive definite Lyapunov function

$$
V(x, y)=x^{4}+y^{4} .
$$

The derivative of this Lyapunov function is given by

$$
\dot{V}(x, y)=-4 \sin (\theta)\left(x^{6}+y^{6}\right),
$$

which is negative definite for $0<\theta<\pi$. Therefore, when $\theta$ belongs to this range, the origin of (12) is asymptotically stable and the system admits the degree 4 Lyapunov function given in (14). On the other hand, we claim that for $\theta$ small enough, the system cannot admit a degree 6 (homogeneous) polynomial Lyapunov function. To argue by contradiction, we suppose that for arbitrarily small and positive values of $\theta$ the system admits sextic Lyapunov functions $W_{\theta}$. Since the vector field satisfies the symmetry

$$
\left(\begin{array}{c}
\dot{x}(y,-x) \\
\dot{y}(y,-x)
\end{array}\right)=\left(\begin{array}{cc}
0 & 1 \\
-1 & 0
\end{array}\right)\left(\begin{array}{l}
\dot{x} \\
\dot{y}
\end{array}\right)
$$

we can assume that the Lyapunov functions $W_{\theta}$ satisfy the symmetry $W_{\theta}(y,-x)=W_{\theta}(x, y)$. 5 This means that $W_{\theta}$ can be parameterized with no odd monomials, i.e., in the form

$$
W_{\theta}(x, y)=c_{1} x^{6}+c_{2} x^{2} y^{4}+c_{3} x^{4} y^{2}+c_{4} y^{6},
$$

where it is understood that the coefficients $c_{1}, \ldots, c_{4}$ are a function of $\theta$. Since by our assumption $\dot{W}_{\theta}$ is negative definite for $\theta$ arbitrarily small, an argument identical to the one used in the proof of Theorem 3.1 implies that as $\theta \rightarrow 0$, $W_{\theta}$ converges to a nonzero sextic homogeneous polynomial $W_{0}$ whose derivative $\dot{W}_{0}$ along the trajectories of (13) (with $\theta=0$ ) is non-positive. However, note that when $\theta=0$, the trajectories of (13) go on periodic orbits tracing the level sets of the function $x^{4}+y^{4}$. This implies that $\dot{W}_{0}=\frac{\partial W_{0}}{\partial x} y^{3}+$ $\frac{\partial W_{0}}{\partial y}\left(-x^{3}\right)=0$. If we write out this equation, we obtain

$\dot{W}_{0}=\left(6 c_{1}-4 c_{2}\right) x^{5} y^{3}+2 c_{2} x y^{7}-2 c_{3} x^{7} y+\left(4 c_{3}-6 c_{4}\right) x^{3} y^{5}=0$,

which implies that $c_{1}=c_{2}=c_{3}=c_{4}=0$, hence a contradiction.

Remark 4.1: We have numerically computed the range $0<\theta<0.0267$, for which the conclusion of Theorem 4.1 holds. This bound has been computed via sum of squares relaxation and semidefinite programming (SDP) by using the SDP solver SeDuMi [41]. What allows the search for a Lyapunov function for the vector field in (13) to be exactly cast as a semidefinite program is the fact that all nonnegative bivariate forms are sums of squares.

\section{ACKNOWLEDGEMENTS}

I would like to express my gratitude to my advisor, Pablo Parrilo, for his very fruitful comments and suggestions on this paper.

\footnotetext{
${ }^{5}$ To see this, note that any Lyapunov function $V_{\theta}$ for this system can be made into one satisfying this symmetry by letting $W_{\theta}(x, y)=V_{\theta}(x, y)+$ $V_{\theta}(y,-x)+V_{\theta}(-x,-y)+V_{\theta}(-y, x)$.
}

\section{REFERENCES}

[1] N. Samardzija. Stability properties of autonomous homogeneous polynomial differential systems. Journal of Differential Equations, 48(1):60-70, 1983.

[2] A. Andreini, A. Bacciotti, and G. Stefani. Global stabilizability of homogeneous vector fields of odd degree. Systems and Control Letters, 10(4):251-256, 1988.

[3] L Grüne. Homogeneous state feedback stabilization of homogeneous systems. In Proceedings of the $39^{\text {th }}$ IEEE Conference on Decision and Control, 2000.

[4] J. Baillieul. The geometry of homogeneous polynomial dynamical systems. Nonlinear analysis, Theory, Methods and Applications, 4(5):879-900, 1980.

[5] M. A. Hammamia and H. Jerbia. The stabilization of homogeneous cubic vector fields in the plane. Applied Mathematics Letters, 7(4):9599, 1994.

[6] L. Rosier. Homogeneous Lyapunov function for homogeneous continuous vector fields. Systems and Control Letters, 19(6):467-473, 1992.

[7] L. Moreau, D. Aeyels, J. Peuteman, and R. Sepulchre. Homogeneous systems: stability, boundedness and duality. In Proceedings of the 14th Symposium on Mathematical Theory of Networks and Systems, 2000.

[8] W. Hahn. Stability of Motion. Springer-Verlag, New York, 1967.

[9] V. I. Arnold. Problems of present day mathematics, XVII (Dynamical systems and differential equations). Proc. Symp. Pure Math., 28(59), 1976.

[10] N. C. A. da Costa and F. A. Doria. On Arnold's Hilbert symposium problems. In Computational Logic and Proof Theory, volume 713 of Lecture Notes in Computer Science, pages 152-158. Springer, 1993.

[11] N. C. A. da Costa and F. A. Doria. Undecidable Hopf bifurcation with undecidable fixed point. International Journal of Theoretical Physics, 33(9), 1994.

[12] E. Hainry. Decidability and undecidability in dynamical systems. Research report. Available at http://hal.inria.fr/inria-00429965/PDF/dynsys.pdf, 2009.

[13] D. S. Graca, J. Buescu, and M. L. Campagnolo. Boundedness of the domain of definition is undecidable for polynomial ODEs. In Proceedings of the Fourth International Conference of Computability and Complexity in Analysis, 2007.

[14] V. D. Blondel, O. Bournez, P. Koiran, and J. N. Tsitsiklis. The stability of saturated linear dynamical systems is undecidable. J. Comput. System Sci., 62(3):442-462, 2001.

[15] V. D. Blondel and J. N. Tsitsiklis. The boundedness of all products of a pair of matrices is undecidable. Systems and Control Letters, 41:135-140, 2000.

[16] V. D. Blondel and J. N. Tsitsiklis. Complexity of stability and controllability of elementary hybrid system. Automatica, 35:479-489, 1999.

[17] V. D. Blondel, O. Bournez, P. Koiran, C. H. Papadimitriou, and J. N. Tsitsiklis. Deciding stability and mortality of piecewise affine systems. Theoretical Computer Science, 255(1-2):687-696, 2001.

[18] O. Bournez and M. L. Campagnolo. A survey on continuous time computations. New Computational Paradigms, 4:383-423, 2008.

[19] E. D. Sontag. From linear to nonlinear: some complexity comparisons. In Proceedings of the $34^{\text {th }}$ IEEE Conference on Decision and Control, 1995.

[20] V. D. Blondel and J. N. Tsitsiklis. Overview of complexity and decidability results for three classes of elementary nonlinear systems. In Learning, Control and Hybrid Systems, pages 46-58. Springer, 1998.

[21] V. D. Blondel and J. N. Tsitsiklis. A survey of computational complexity results in systems and control. Automatica, 36(9):1249$1274,2000$.

[22] R. Jungers and V. D. Blondel. On the finiteness property for rational matrice. Linear Algebra and its Applications, 428:2283-2295, 2008.

[23] P. A. Parrilo. Structured semidefinite programs and semialgebraic geometry methods in robustness and optimization. $\mathrm{PhD}$ thesis, California Institute of Technology, May 2000.

[24] A. Papachristodoulou and S. Prajna. On the construction of Lyapunov functions using the sum of squares decomposition. In IEEE Conference on Decision and Control, 2002.

[25] M. M. Peet. Exponentially stable nonlinear systems have polynomial Lyapunov functions on bounded regions. IEEE Trans. Automat. Control, 54(5):979-987, 2009. 
[26] M. M. Peet and A. Papachristodoulou. A converse sum of squares Lyapunov result with a degree bound. IEEE Trans. Automat. Control, 2011. To appear.

[27] A. A. Ahmadi, M. Krstic, and P. A. Parrilo. A globally asymptotically stable polynomial vector field with no polynomial Lyapunov function. In Proceedings of the $50^{\text {th }}$ IEEE Conference on Decision and Control, 2011.

[28] A. A. Ahmadi and P. A. Parrilo. Converse results on existence of sum of squares Lyapunov functions. In Proceedings of the $50^{\text {th }}$ IEEE Conference on Decision and Control, 2011.

[29] A. A. Ahmadi. Algebraic relaxations and hardness results in polynomial optimization and Lyapunov analysis. $\mathrm{PhD}$ thesis, Massachusetts Institute of Technology, September 2011.

[30] A. Tarski. A decision method for elementary algebra and geometry. University of California Press, Berkeley and Los Angeles, Calif., 1951. Second edition.

[31] A. Seidenberg. A new decision method for elementary algebra. Ann. of Math. (2), 60:365-374, 1954.

[32] Michael R. Garey and David S. Johnson. Computers and Intractability. W. H. Freeman and Co., San Francisco, Calif., 1979.

[33] K. G. Murty and S. N. Kabadi. Some NP-complete problems in quadratic and nonlinear programming. Mathematical Programming, 39:117-129, 1987.

[34] E. de Klerk and D. V. Pasechnik. Approximation of the stability number of a graph via copositive programming. SIAM Journal on Optimization, 12(4):875-892, 2002.

[35] B. Reznick. Some concrete aspects of Hilbert's 17th problem. In Contemporary Mathematics, volume 253, pages 251-272. American Mathematical Society, 2000.

[36] H. Khalil. Nonlinear systems. Prentice Hall, 2002. Third edition.

[37] A. A. Ahmadi and P. A. Parrilo. On higher order derivatives of Lyapunov functions. In Proceedings of the 2011 American Control Conference, 2011.

[38] P. A. Parrilo. Semidefinite programming relaxations for semialgebraic problems. Mathematical Programming, 96(2, Ser. B):293-320, 2003.

[39] T. S. Motzkin. The arithmetic-geometric inequality. In Inequalities (Proc. Sympos. Wright-Patterson Air Force Base, Ohio, 1965), pages 205-224. Academic Press, New York, 1967.

[40] J. Löfberg. Yalmip : A toolbox for modeling and optimization in MATLAB. In Proceedings of the CACSD Conference, 2004. Available from http: //control.ee.ethz.ch/ joloef/yalmip.php.

[41] J. Sturm. SeDuMi version 1.05, October 2001. Latest version available at http://sedumi.ie.lehigh.edu/.

[42] J. Nie, J. Demmel, and B. Sturmfels. Minimizing polynomials via sum of squares over the gradient ideal. Mathematical Programming, 106(3, Ser. A):587-606, 2006.

[43] A. Bacciotti and L. Rosier. Liapunov Functions and Stability in Control Theory. Springer, 2005. 